CASE REPORT

R. Shah

J. Markert

A.K. Bag

J.K. Curé

\title{
Diffusion Tensor Imaging in Hypertrophic Olivary Degeneration
}

SUMMARY: We report DTI and fiber tractography findings in a case of hypertrophic olivary degeneration. A 51-year-old man presented with an abnormal gait and visual difficulties. MR imaging showed enlargement of the right medullary olive and a vascular lesion in the right pontine tegmentum. Fiber tractography showed decreased volume of the right central tegmental tract, supporting a diagnosis of HOD.

ABBREVIATIONS: HOD = hypertrophic olivary degeneration; DTI = diffusion tensor imaging

H OD is a degenerative disorder of the inferior olivary nucleus that occurs after an insult to the dentatorubro-olivary pathway. Common clinical findings associated with HOD include palatal myoclonus, dentatorubral tremor, and ocular myoclonus. A lesion of the ipsilateral brain stem or contralateral cerebellum that disrupts the Guillian-Mollaret triangle is thought to be responsible. We present a case of hypertrophic olivary degeneration in which demonstration of a decrease in the volume of the central tegmental tract by DTI and fiber tractography contributed to the diagnosis.

\section{Case Report}

A 51-year-old man developed new-onset dizziness, balance problems, and visual and gait difficulty. A neurologic examination revealed ataxia and left-sided dysmetria. There was bilateral rotary nystagmus to the right side and no palatal myoclonus.

MR imaging of the brain and DTI were performed on a TwinSpeed HDxt 1.5T scanner (GE Healthcare, Milwaukee, Wisconsin). T2-weighted and fluid-attenuated inversion recovery images revealed focal hyperintensity and expansion of the right medullary olive. There was no enhancement in this region following contrast administration and no loss of signal intensity in this lesion on the susceptibilityweighted sequence. A small enhancing vascular lesion in the right pontomesencephalic tegmentum showed hypointensity on a susceptibility-weighted sequence (Fig 1).

DTI was performed by using a Stejskal-Tanner sequence with single-shot spin-echo echo-planar imaging (TR/TE, 8500/104.7 ms; matrix size, $128 \times 128$; FOV, $26 \times 20.8 \mathrm{~cm}$; and section thickness, 5.0 $\mathrm{mm})$. We obtained diffusion-weighted images along 25 different directions with a b-value of $1000 \mathrm{~s} / \mathrm{mm}^{2}$, as well as an image without diffusion weighting $\left(b=0 \mathrm{~s} / \mathrm{mm}^{2}\right)$. Brain fiber tracking was performed by using software Version 4.3 on an Advantage Windows workstation (GE Healthcare). Seed regions of interest were placed on both red nuclei, and fiber tractography maps were generated. Fractional anisotropy values were calculated by placing identical regions of interest on the central tegmental tract at the level of pons and medullary olives bilaterally.

Received August 4, 2009; accepted September 11

From the Department of Diagnostic Radiology (R.S., A.K.B., J.K.C.), Division of Neuroradiology, and Department of Surgery (J.M.), Division of Neurosurgery, University of Alabama at Birmingham, Birmingham, Alabama

Please address correspondence to Ritu Shah, MD, Assistant Professor in Neuroradiology, University of Alabama Department of Radiology, 619 19th St South, Birmingham, AL 35249-6830; e-mail: ritushah@uabmc.edu

DOI 10.3174/ajnr.A1911
There was decreased volume of the central tegmental tract ipsilateral to the medullary lesion without deviation, deformation, or interruption. The fractional anisotropy value was slightly decreased in the right pontine tegmentum ( 0.412 on the right versus 0.455 on the left). There was also nonvisualization of the decussation of the right superior cerebellar peduncle on fiber tractography (Fig 2).

\section{Discussion}

Hypertrophic olivary degeneration is a form of trans-synaptic degeneration with hypertrophy rather than atrophy of the inferior olivary nucleus in response to neurologic insult to the dentatorubro-olivary pathway. ${ }^{1}$ These dentatorubral olivary connections were first described by Guillian and Mollaret in 1931 in association with oculopalatal tremor and HOD. ${ }^{2}$ This pathway, known as the Guillian-Mollaret triangle, connects the dentate nucleus of the cerebellum, the red nucleus of the midbrain, and the inferior olivary nucleus of the medulla. Fibers from the dentate nucleus ascend to the contralateral red nucleus in the superior cerebellar peduncle. These fibers decussate within the brachium conjunctivum and synapse in the contralateral red nucleus. The fibers from the red nucleus descend in the central tegmental tract to the inferior olivary nucleus. The olive then sends fibers to the contralateral dentate nucleus via the inferior cerebellar peduncle, completing the triangle. Lesions that disrupt the afferent pathways to the olive (dentatorubral and rubro-olivary pathways) result in HOD, but lesions of efferent pathways are less likely to cause HOD. Thus, lesions in contralateral cerebellum and superior cerebellar peduncle or the ipsilateral brain stem involving the red nucleus and central tegmental tract are likely to result in HOD, whereas lesions of inferior cerebellar peduncle do not cause HOD.

Infarcts, hemorrhages, traumatic brain injury, tumor, and surgery for vascular lesions have all been reported as causes of HOD. ${ }^{3-5}$ Diagnosis of HOD on MR imaging is made by the presence of a T2-hyperintense nonenhancing olivary lesion in association with another lesion in the contralateral dentate nucleus, contralateral superior cerebellar peduncle, ipsilateral red nucleus, or ipsilateral pontine tegmentum.

In our patient, a small capillary telangiectasia was present in the pontine tegmentum ipsilateral to the medullary lesion. There was no evidence of prior hemorrhage and no past history of surgery. Capillary telangiectasias are usually incidental findings on MR imaging ${ }^{6}$ and have not been previously reported as a cause of HOD. Therefore, the findings on conven- 

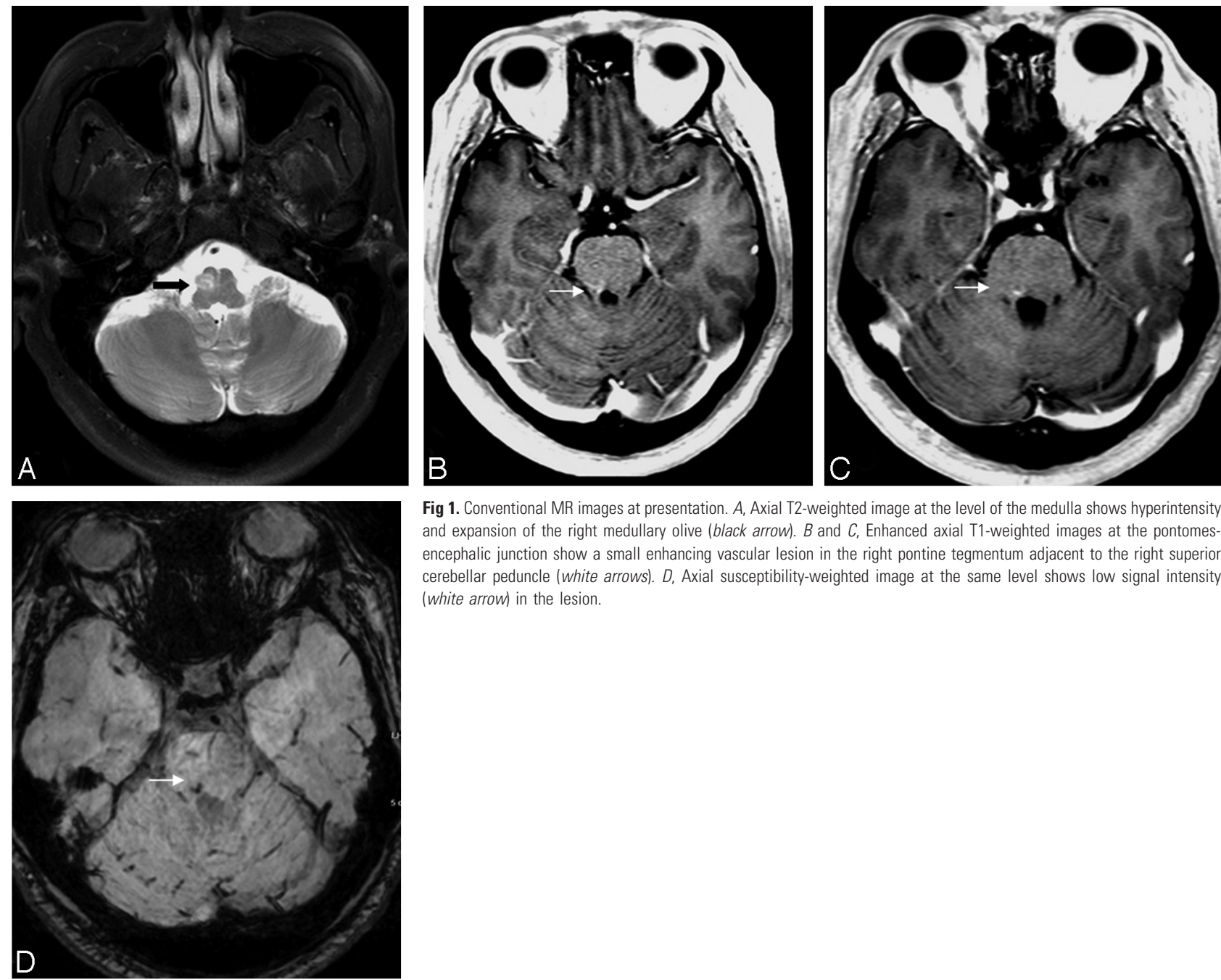

Fig 1. Conventional MR images at presentation. A, Axial T2-weighted image at the level of the medulla shows hyperintensity and expansion of the right medullary olive (black arrow). $B$ and $C$, Enhanced axial T1-weighted images at the pontomesencephalic junction show a small enhancing vascular lesion in the right pontine tegmentum adjacent to the right superio cerebellar peduncle (white arrows). D, Axial susceptibility-weighted image at the same level shows low signal intensity (white arrow) in the lesion.

tional MR imaging were not conclusive. On fiber tractography, we found decreased central tegmental tract volume away

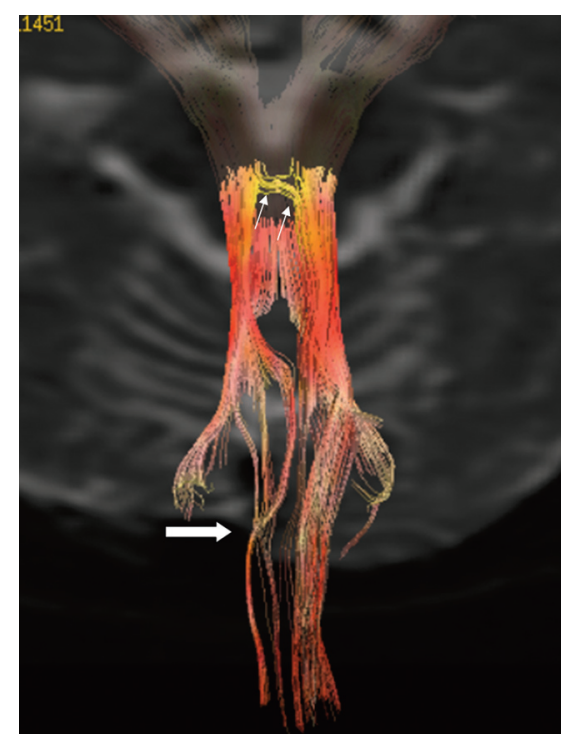

Fig 2. Fiber tractography obtained after placing seed regions of interest on the red nuclei. The right central tegmental tract is thinned (thick white arrow). Decussating dentatorubral fibers of the left superior cerebellar peduncle are seen going from left to right (small white arrows), but the right superior cerebellar decussation is not visualized. from the site of the inferior olivary lesion in this case, which was consistent with tract "degeneration" per criteria developed by Lazar et al. ${ }^{7}$ There was no deviation, deformation, or interruption of tracts as has been described with brain stem tumors and masses. ${ }^{8}$

Normal anatomy of white matter tracts in the brain stem has been described by several authors on $3 \mathrm{~T}$ and $1.5 \mathrm{~T}$ systems. ${ }^{9,10}$ Central tegmental tracts demonstrated by high-resolution DTI are symmetric and run in a superior-inferior direction. ${ }^{9}$ Decussation of superior cerebellar peduncles is identified at the midbrain level as transversely oriented fibers. ${ }^{10}$ While we were able to visualize the decussating fibers of the superior cerebellar peduncle from left to right on tractography in our patient, the right superior cerebellar decussation was not seen. This was interesting because the right superior cerebellar peduncle and its decussation form a part of the contralateral Guillain-Mollaret pathway. A likely explanation for these findings lies in the location of the capillary telangiectasia, which involved both the right central tegmental tract and the decussating fibers of the right superior cerebellar peduncle. We speculate that a similar line of reasoning could also potentially explain the occurrence of bilateral HOD in the presence of a unilateral lesion at the pontomesencephalic junction, as previously reported by Tsui et al ${ }^{11}$ and in 1 of the patients in the series by Hornyak et al. ${ }^{4}$ 
In conclusion, DTI and fiber tractography can demonstrate the disruption of pathways involved in pathogenesis of hypertrophic olivary degeneration and are useful in establishing the diagnosis when findings on conventional MR imaging are equivocal.

\section{References}

1. Oppenheim $\mathrm{H}$. Uber oliven degeneration bei atheromatose der basalen hin arterien. Berl Klin Wochenschr 1887;34:638-39

2. Guillian G, Mollaret P. Deux cas de myoclonies synchrones et rhythmes velopharyngo-laryngo-oculo-diaphragmatiques. Rev Neurol 1931;2:545-66

3. Goyal M, Versnick E, Tuite $P$, et al. Hypertrophic olivary degeneration: metaanalysis of the temporal evolution of MR findings. AJNR Am J Neuroradiol 2000;21:1073-77

4. Hornyak M, Osborn AG, Couldwell WT. Hypertrophic olivary degeneration after surgical removal of cavernous malformations of the brain stem: report of four cases and review of the literature. Acta Neurochir (Wien) 2008; 150:149-56

5. Kitajima M, Korogi Y, Shimomura O, et al. Hypertrophic olivary degeneration: MR imaging and pathologic findings. Radiology 1994;192:539-43

6. Rabinov JD. Diagnostic imaging of angiographically occult vascular malformations. Neurosurg Clin N Am 1999;10:419-32

7. Lazar M, Alexander AL, Thottakara PJ, et al. White matter reorganization after surgical resection of brain tumors and vascular malformations. AJNR Am J Neuroradiol 2006;27:1258-71

8. Chen X, Weigel D, Ganslandt O, et al. Diffusion tensor imaging and white matter tractography in patients with brainstem lesions. Acta Neurochir (Wien) 2007;149:1117-31

9. Nagae-Poetscher LM, Jiang H, Wakana S, et al. High-resolution diffusion tensor imaging of the brain stem at 3T. AJNR Am J Neuroradiol 2004;25:1325-30

10. Salamon N, Sicotte N, Alger J, et al. Analysis of the brain-stem white-matter tracts with diffusion tensor imaging. Neuroradiology 2005;47:895-902

11. Tsui EY, Cheung YK, Mok CK, et al. Hypertrophic olivary degeneration following surgical excision of brainstem cavernous hemangioma: a case report. Clin Imaging 1999;23:215-17 